Short reports

\title{
Subarachnoid haemorrhage: difficulties in diagnosis and treatment
}

\author{
S D Johnston, T J Robinson
}

\begin{abstract}
Summary
Aneurysmal subarachnoid haemorrhage is associated with a uniquely severe headache of acute onset. Classical cases are readily identified as such, although this is not always the case. Four cases who were admitted to a district general hospital within a 3-month period are presented, because they demonstrate a variety of presentations, management options, and outcomes.
\end{abstract}

Keywords: subarachnoid haemorrhage; aneurysm; lumbar puncture

Subarachnoid haemorrhage is usually the result of rupture of an intracranial aneurysm. It can be a devastating event since $12 \%$ of patients die before receiving medical treatment, ${ }^{1} 40 \%$ of hospitalised patients die in the first month after the event, ${ }^{2}$ and more than one-third of those who survive have a major neurological deficit. ${ }^{2}$ Indeed, the case fatality for subarachnoid haemorrhage has not changed in recent years, despite advances in diagnosis and treatment of this condition..$^{2-4} \mathrm{~A}$ 'warning leak' may occur in up to one half of patients although this is often misdiagnosed as migraine or sinusitis. We report four cases who presented to a district general hospital within a 3-month period, since they demonstrate a range of possible presentations, management options and outcomes as a consequence of aneurysmal subarachnoid haemorrhage.

\section{Case reports}

A 43-year-old man presented with sudden onset of occipital headache, neck pain, diarrhoea and vomiting, and generalised aches and pains. He had a previous history of migraine, although he described this as a different kind of headache. His two daughters also had headache, diarrhoea, and flu-like symptoms. Computed tomography (CT) brain scan, carried out 6 days following admission, revealed blood in the right sylvian fissure and prominence of the lateral ventricles. Lumbar puncture confirmed the presence of xanthochromia. The initial cerebral angiogram was normal, although a second angiogram revealed a right posterior communicating artery aneurysm. $\mathrm{He}$ subsequently underwent craniotomy and clipping of the aneurysm. He recovered fully apart from a residual partial right third nerve palsy.
A 76-year-old woman presented with a 3-hour history of headache and backache. Prior to admission she had been treated by her general practitioner for a chest infection and during a fit of coughing she experienced a sudden onset of pain across her back, accompanied by sweating, nausea and occipital headache. Following admission she became more drowsy and developed a right-sided ptosis and a dilated left pupil. CT brain scan indicated extensive bilateral subarachnoid haemorrhage. Despite intravenous nimodipine she deteriorated and died 6 days following admission. No cerebral angiography was performed.

A 39-year-old man was admitted with a 5-day history of headache which occurred during an unaccustomed 20-mile cycle ride. The headache was bifrontal and throbbing. The working diagnosis was exercise-induced migraine but since his headache failed to settle after 3 days a CT brain scan was performed which was normal. No lumbar puncture was performed and he was discharged. One week following discharge he was re-admitted with acute onset headache and a rapid reduction in level of consciousness until he became unresponsive to painful stimuli. $\mathrm{He}$ required intubation and assisted ventilation. CT brain scan revealed massive subarachnoid haemorrhage with intracerebral extension also involving the left lateral ventricle and third ventricle. Seven days following admission a repeat CT brain scan indicated obstructive hydrocephalus and he was transferred to the regional neurosurgical unit. He failed to make any significant progress despite a ventricular shunt and he died 13 days later.

A 31-year-old woman presented one week following the birth of her healthy baby girl. The pregnancy was uncomplicated and she had no previous history of pre-eclampsia. She complained of a gradual onset of headache which had been present for the previous 20 hours, occurring initially in the neck and occiput, then spreading to the right temporoparietal region. Following admission to the ward she collapsed in the toilet but was subsequently able to co-operate with her admission interview and examination. Her headache subsequently responded dramatically to subcutaneous sumatriptan within 10 minutes. There were no abnormal neurological signs although she had a bradycardia ( 44 beats $/ \mathrm{min}$ ) and became more vacant a few hours following admission. The following day she became incontinent of urine and had a tonic-clonic seizure. CT brain scan revealed a large subarachnoid haemorrhage 
with intraventricular extension and generalised oedema. Following transfer to the regional neurosurgical unit a ventricular drain was inserted and although the cerebrospinal fluid was blood-stained it was not under pressure. This was later complicated by ventriculitis. Cerebral angiogram revealed marked spasm of the intracranial vessels and the possibility of a left posterior communicating artery aneurysm. A repeat CT brain scan indicated a degree of hydrocephalus and a ventriculo-peritoneal shunt was inserted. Her condition did not improve and she continued to be disorientated and had a persistent vacant expression. A percutaneous endoscopic gastrostomy tube was inserted for feeding and she was transferred to a long-stay hospital for continuing nursing care.

\section{Discussion}

This series of cases serves to highlight a number of lessons with regard to the presentation and management of subarachnoid haemorrhage. Headache may not be the sole or predominant symptom as demonstrated by the first two cases. As in the third case, the onset of headache may be associated with exertion or stress, although it is recognised that it may occur at any time. ${ }^{5}$ This headache may have represented a 'warning leak' which can occur in one third to one half of patients, preceding the haemorrhage by several days or weeks. ${ }^{6}$

While CT brain scanning is a very sensitive test at detecting subarachnoid haemorrhage with a sensitivity of $90-95 \%$ if performed within 24 hours, this falls to $80 \%$ if the scan is performed at 3 days. ${ }^{7}$ The third case reinforces the fact that a normal CT brain scan does not rule out a subarachnoid haemorrhage and a lumbar puncture looking for xanthochromia and bilirubin degradation products by spectrophotometry must be performed to confidently exclude the diagnosis. ${ }^{8}$ Lumbar puncture must be performed after 12 hours has elapsed in order to exclude a subarachnoid haemorrhage,

1 Schievink WI, Wijdicks EFM, Parisi JE, Piepgras DG Whisnant JP. Sudden death from aneurysmal subarachnoid haemorrhage. Neurology 1995;45:871-4.

2 Fogelholm R, Hernesniemi J, Vapalahti M. Impact of early surgery on outcome after aneurysmal subarachnoid haemorrhage: a population based study. Stroke 1993;24: 1649-54.

3 Inagawa T, Tokuda $\mathrm{Y}$, Ohbayashi N, Takaya M, Moritake K. Study of aneurysmal subarachnoid haemorrhage in Izumo City, Japan. Stroke 1995;26:761-7.

4 Schievink WI, Wijdicks EFM, Piepgras DG, Chu C-P O'Fallon WM, Whisnant JP. The poor prognosis of ruptured intracranial aneurysms of the posterior circulation. 7 Neurosurg 1995;82:791-5.

5 Schievink WI. Karemaker JM, Hageman LM, van der Werf DJM. Circumstances surrounding aneurysmal subarachnoid haemorrhage. Surg Neurol 1988;32:266-72.

\section{Learning points}

- subarachnoid haemorrhage may be a difficult diagnosis to make

- the classical presentation is of a uniquely severe headache of acute onset

- a suggestive history must always be investigated by CT brain scan and lumbar puncture (for bilirubin degradation products by

spectrophotometry), if the brain scan is negative

- headache may not be the sole or predominant symptom

since prior to this a false negative result may be obtained. Lumbar puncture is contraindicated when there is clinical evidence of raised intracranial pressure, papilloedema or when an intracranial space-occupying lesion is suspected. Lumbar puncture is a safe procedure providing proper techniques and indications are followed. Complications may occur and include postdural puncture headache, nerve root irritation, low back pain, infections, bleeding complications, and cerebral and spinal herniation. ${ }^{9}$ As in the final case, it is recognised that labour and delivery increase the risk of spontaneous subarachnoid haemorrhage. ${ }^{10} \mathrm{It}$ is clear from the last case that of those who do survive the initial haemorrhage many are left with major neurological deficit or persistent cognitive deficit. ${ }^{23}$

In summary, the first step in the diagnosis of aneurysmal subarachnoid haemorrhage is to consider its possibility in any patient with a uniquely severe headache of acute onset. It is then necessary to investigate appropriately and thoroughly with a CT brain scan, followed by lumbar puncture if the CT scan is negative, in order to make a rapid diagnosis and allow appropriate treatment to be given in specialised regional centres. This case series illustrates that failure to do so can have devastating consequences for the patient.

6 Ostergard JR. Headache as a warning symptom of impending aneurysmal subarachnoid haemorrhage. Cephalgia 1991;11:53-5.

7 Van Gijn J, van Dongen KJ. The time course of aneurysmal haemorrhage on computed tomograms. Neuroradiology 1982;23:153-6.

8 Findlay JM. Current management of aneurysmal subarachnoid haemorrhage guidelines from the Canadian Neurosurgical Society. Can $\mathcal{F}$ Neurol Sci 1997:24;161-70.

9 Evans RW. Complications of lumbar puncture. Neurol Clin 1998;16:83-105.

10 Barno A, Freeman DW. Maternal deaths due to spontaneous subarachnoid hemorrhage. Am $\mathcal{f}$ Obstet Gynecol 1976;125:384-92. 